\title{
TRANSFORMACIONES SOCIODEMOGRÁFICAS DE LA ÉLITE PARLAMENTARIA EN VENEZUELA (1993-1998)
}

\author{
Marisa Ramos* y Flavia Freidenberg**
}

\section{INTRODUCCIÓN}

El objeto de análisis de este artículo es el estudio de la estructura sociológica de la élites parlamentarias en Venezuela en el período 1993-1998, comparándola con las de la actual legislatura. El análisis de la élite parlamentaria enlaza con dos campos de estudio que han sido redimensionados durante la última década en el campo de las ciencias sociales: el del papel de los poderes legislativos en las democracias actuales y el de las élites políticas. En cuanto al primer caso, se está en camino de poner fin al relativo abandono de análisis de carácter general sobre los parlamentos, profundizado por la tradicional situación latinoamericana en la que los poderes legislativos apenas han desempeñado un papel político relevante. Sin embargo, la ola democratizadora de la década de 1980 vino acompañada de una generalizada conformación de parlamentos, situación que instala en la agenda política la reivindicación y fortalecimiento de su papel (Close, 1995).

Por otra parte, también el estudio de las élites políticas ha tomado un nuevo vigor en tanto tema susceptible de constituirse en variable explicativa de los procesos de consolidación democrática (Diamond, 1993; Higley y Gunther, 1992; Linz y Stepan, 1997). En este sentido, cuestiones tales como la continuidad o discontinuidad de las élites en los periodos de transición, el consenso o conflicto en la interacción entre las ellas, su homogeneidad o heterogeneidad o el grado de coincidencia de las mismas con la sociedad a la que representan en tanto espejo de la misma se han convertido en líneas analíticas importantes al momento de estudiar los sistemas políticos.

Desde esta última perspectiva, los enfoques neoelitistas tratan de averiguar hasta qué punto las élites políticas tienen vinculaciones con otras élites como las económicas o culturales y en qué medida representan las tendencias sociodemográficas de su sociedad. Estos estudios ponen énfasis en el peso de los elementos de la estructura social en la composición de las Cámaras (Alcántara y Llamazares, 1997: 18). De alguna manera, implica plantearse cuál es la relación entre características sociales de las élites y la defensa de determinados intereses sociales o, planteado de otra manera, conocer la vinculación entre la defensa de ciertos intereses y los grupos sociales de los que proceden estas éli- tes. Este enfoque supone que las características de la clase política se relacionan con los rasgos de la estructura social, por lo que la representación puede darse como espejo de esa realidad social o puede ser solo representativa de un grupo minoritario de la sociedad.

En este sentido, este análisis va a intentar contrastar hipótesis clásicas, al estilo de las recogidas por Putnam (1976) y Von Beyme (1995) sobre la relación entre determinadas características sociodemográficas y la élite política. La confirmación o rechazo de estas hipótesis dará la medida de la renovación de esta y su grado de homogeneidad e integración en tanto élite.

Si bien Venezuela no es un caso representativo de nuevo régimen democrático, ya que ha funcionado ininterrumpidamente como tal desde 1958, sí existen una serie de elementos que permiten plantear la existencia de una importante ruptura en la composición de la élite parlamentaria, constituyendo las dos últimas legislaturas un punto de inflexión claro en el desarrollo político venezolano. Tal como expresan otros artículos de este mismo número, la composición del Congreso de la legislatura anterior y de la actual reflejan la ruptura definitiva del sistema de partidos que venía funcionando desde 1958, no sólo en el sentido de que se haya acabado con el sistema bipartidista, sino que el multipartidismo ha pasado de ser, en los términos de Sartori (1976), limitado en 1993 a extremo en 1998. Los dos partidos tradicionales y mayoritarios hasta 1993 -Acción Democrática (AD) y COPEI- perdieron su hegemonía con la entrada en escena de Convergencia (Conven) y el crecimiento de Causa $\mathrm{R}$ (CR). Estos hechos fueron el primer paso en ese proceso de ruptura del sistema de partidos y supusieron una importante renovación de la Cámara de Diputados, que quedó integrada por:

- Acción Democrática, con 55 diputados, de origen socialdemócrata, aunque fuertemente desdibujado ideológicamente desde hacía un tiempo;

\footnotetext{
Profesora Titular del Área de Ciencia Política y de la Administración de la Universidad de Salamanca.

** Investigadora del Instituto de Estudios de Iberoamérica y Portugal de la Universidad de Salamanca.
} 
- COPEI, con 53 diputados, de corte demócrata-cristiano;

- Causa R, con 40 diputados, que surge de una escisión del Partido Comunista de Venezuela (PCV) y con orígenes sindicales;

- Convergencia, con 26 diputados, creado para cobijar al presidente Caldera cuando decidió abandonar COPEI y que puede considerarse una escisión de éste; y

- Movimiento Al Socialismo (MAS), con 24 diputados, también surgido de una escisión del PCV, de corte socialista, aunque también inmerso en un proceso de redefinición ideológica.

El segundo paso en ese proceso de ruptura del sistema de partidos encuentra su mayor expresión en los comicios de noviembre de 1998, a partir de los cuales se ha transformado de manera radical la correlación de fuerzas dentro de la Cámara, que ha quedado integrada por

- Polo Patriótico, 75 diputados, de los cuales 46 corresponden al Movimiento Quinta República (MVR), una agrupación creada sobre la base de una organización política de composición cívico-militar llamada Movimiento Bolivariano Revolucionario 200 (MBR200) y que se convierte en un partido político para promocionar la candidatura presidencial de Hugo Chávez Frías; 18 diputados pertenecen al MAS; 7 son miembros de Patria para Todos (PPT), una escisión de Causa $\mathrm{R}$ articulada detrás de la figura del exgobernador y líder sindical Andrés Velásquez, quien fuera candidato presidencial de Causa $\mathrm{R}$ para las elecciones de 1993, y el resto forma parte de agrupaciones menores (4 diputados). El contenido del discurso de este frente es principalmente nacionalista, antineoliberal y popular;

- Acción Democrática, 62 diputados,

- COPEI, 27 diputados,

- Causa R, 6 diputados,

- Convergencia, 5 diputados,

- Proyecto Venezuela (PROVEN), 20 diputados provenientes de la centroderecha, creado para promocionar la candidatura de Salas Römer a la presidencia de la República.

La fuente principal de información para este análisis ha sido una encuesta que se ha realizado a los parlamentarios venezolanos en la legislatura del período 1993-1998. La encuesta administrada a los diputados se inserta en un proyecto más amplio que se ha aplicado a la mayoría de los países latinoamericanos ${ }^{\prime}$. En el caso venezolano el cuestionario se realizó durante los meses de marzo y abril de 1995 a una muestra de 69 diputados representativa por partido político.

La información referente a los diputados de la legislatura de 1998 tiene un origen distinto. Sobre la base de la realización de preguntas similares al cuestionario antes descrito $^{2}$, el periódico El Universal realizó una encuesta de extensión reducida a todos los parlamentarios de la actual Cámara ${ }^{3}$. Si bien hay preguntas que siendo idénticas ofrecen valores distintos, se ha considerado que el contraste podría ser de sumo interés, aun conociendo las dificultades para la comparación de los datos.

\section{COMPOSICIÓN DE LA ÉLITE PARLAMENTARIA VENEZOLANA}

Putnam (1976) y Von Beyme (1995) apuntan una serie de consideraciones que pueden ser analizadas en tanto hipótesis de la posible relación existente entre la estructura socioeconómica y demográfica y la élite política. Se va a comprobar hasta qué punto en el caso de los parlamentarios venezolanos de la legislatura 1993-1998 se confirman estas hipótesis. En aquellas preguntas donde se cuenten con los datos de la actual legislatura se realizará la comparación.

\section{- Clase social}

Putnam (1976: 21-22) apunta dos modelos de análisis de las élites, el modelo de la independencia y el modelo de la aglutinación. En el primero se plantea que el estrato socioeconómico no es un determinante para formar parte de este grupo político, en tanto que en el segundo modelo se parte de la base de la existencia de una correlación entre el lugar individual en la estratificación política y el lugar en la jerarquía social, es decir, que los sectores económicamente privilegiados monopolizan el liderazgo político. El mismo Putnam señala que las teorías clásicas sobre élites se han decantado por este segundo modelo. Al respecto, indica la evidencia que produce la siguiente generalización: “... in each nation, bigher status ocupation are vastly overrepresented and lower status occupations vastly underrepresented..." (1976: 22)4. En tanto la condición de diputado puede ser equiparada a la pertenencia a un status elevado dentro de la clase política, según esta hipótesis básica de Putnam, la élite parlamentaria debería pertenecer a la clase social alta.

Sin embargo, Von Beyme sin abandonar esta fuerte correlación entre clase social y clase política, introduce el elemento de la representatividad social de la clase política y la novedad que supuso la entrada en los parlamentos de partidos de izquierda. Señala, para el caso de las democracias europeas en la época de entreguerras, el cambio que produce la entrada en los parlamentos de representantes de izquierda pertenecientes a lo que llama la contraélites, es

1. Nos referimos a la investigación de "Élites Parlamentarias en América Latina", que se ha desarrollado en el Instituto de Estudios de Iberoamérica y Portugal de la Universidad de Salamanca, dirigida por Manuel Alcántara Sáez y financiada por la Comisión Interministerial de Ciencia y Tecnología de España (SEC95-0845).

2. Algunas de las preguntas fueron realizadas de manera idéntica a las de la investigación española con el objeto de poder llevar a cabo la comparación de los datos obtenidos.

3. Las entrevistas fueron realizadas a 203 de los 207 diputados venezolanos y a 50 senadores, a través de la vía telefónica o personalmente por reporteros de la redacción o corresponsales en los estados del Diario El Universal. El trabajo de campo se realizó desde el 15 de noviembre de 1998 hasta el 16 de enero de 1999.

4. “...en cada nación, las ocupaciones de alto status son enormemente sobrerepresentadas y las ocupaciones de bajo status enormemente subrepresentadas...”. 
decir, a la clase política que representa a los sectores sociales bajos. Esto genera una diferenciación social en los parlamentarios, con un equilibrio asimétrico: “... las élites burguesas mantuvieron el predominio en el Estado, pero la élite de la clase trabajadora desarrolló su posición de veto..." (1995:108). En suma, se trataría de verificar hasta qué punto se sostiene la hipótesis de Putnam de la equiparación entre los parlamentarios y clase social alta o si el caso venezolano se adecuaría más al señalado por Von Beyme, es decir, una correspondencia entre partidos conservadores o de derecha y clase social alta, y partidos de izquierda y clase social baja.

La diferencia entre los partidos de izquierda y derecha, según las consideraciones más extendidas, serían las siguientes: COPEI estaría considerado como un partido más conservador o de derecha, y AD, Causa R y MAS como partidos de izquierda o centro-izquierda. Según los datos proporcionados por la Investigación de Élites Parlamentarias en América Latina para la Cámara de 1993, los diputados de COPEI ubican a su partido en una posición de centro, siendo la media de 5,7 en una escala de diez gradaciones, donde 1 era izquierda y 10 derecha, y el resto de diputados ubican a sus partidos en la centro izquierda del espectro ideológico. Los legisladores de AD colocan a su partido en una posición de 4,5, los de Causa Radical en 4,6, los de Convergencia en una posición de 4,7 y los del MAS en 3,7; la ubicación más a la izquierda del conjunto de partidos. Ahora bien, este etiquetado no significa que se desconozca el fuerte proceso de desdibujamiento ideológico que en la última década han sufrido los partidos venezolanos, situación agravada en los tres últimos años por el surgimiento de nuevas formaciones políticas de difícil ubicación ideológica. En la legislatura de 1998 y a partir de la utilización de una escala de cinco gradaciones, donde 1 era izquierda y 5 derecha, los diputados de $\mathrm{AD}$ ubicaron a su partido en una posición media de 2,53 y los de Causa $\mathrm{R}$ en 2,25 , lo que podría considerarse como espacios de la centro-izquierda; los del MAS en 1,74, los de PPT en 1,50, los de MVR en 1,71, lo que sería la izquierda; en tanto los diputados de COPEI lo ubicaron en 2,89, los de Convergencia en 2,80 y los de Proyecto Venezuela en 2,76; siendo estas últimas las que se encuentran más hacia la derecha del espectro ideológico.

\begin{tabular}{||l|c|c||}
\hline \multicolumn{3}{||l||}{ Cuadro I: Clase Social de Origen (\%) } \\
\hline & 1993 & 1998 \\
\hline Baja & 11,8 & 22.1 \\
\hline Media-Baja & 64,7 & 28.1 \\
\hline Media & $*$ & 37.0 \\
\hline Media-Alta & 13,8 & 9.0 \\
\hline Alta & 3,1 & 3.6 \\
\hline N & 65 & 261 \\
\hline$*$ En la encuesta de 1993 no se incluía este valor \\
\hline Fuente: Investigación Élites Parlamentarias en América Latina y El Universal \\
\hline
\end{tabular}

Los indicadores utilizados para contrastar estas hipótesis han sido las preguntas en las que se les pedía a los diputados que señalaran su clase social de origen y su nivel de ingresos anuales. En cuanto a su clase social de origen, parece que esta variable puede ser más fidedigna para conocer la clase social a la que pertenece el diputado. Con respecto a la información que brinda para la legislatura de 1993, no se confirmaría la hipótesis de que la mayoría de los legisladores procede de una clase social media-alta o alta: la mayoría $(64,7)$ dijo proceder de una familia de clase social mediabaja, frente a un $13,8 \%$ que señaló su pertenencia a una clase social media-alta y sólo un 3\% dijo proceder de una clase alta. Con relación a la Cámara de 1998, tampoco se confirmaría la hipótesis que asocia a los diputados con la clase alta: una parte importante de los legisladores $(37 \%)$ señaló que su clase social de origen era la clase media; un $28,1 \%$ indicó que pertenecía a una clase media-baja y el 22,1\% se manifestó por la clase social baja. Sólo el 3,6\% dijo proceder de la clase alta. De este modo, en el Congreso venezolano no se corroboraría la hipótesis del modelo de aglutinación presentado por Putnam, sino que abonaría lo sostenido en el modelo de la independencia acerca de la inexistencia de relación entre posición socioeconómica y élite política.

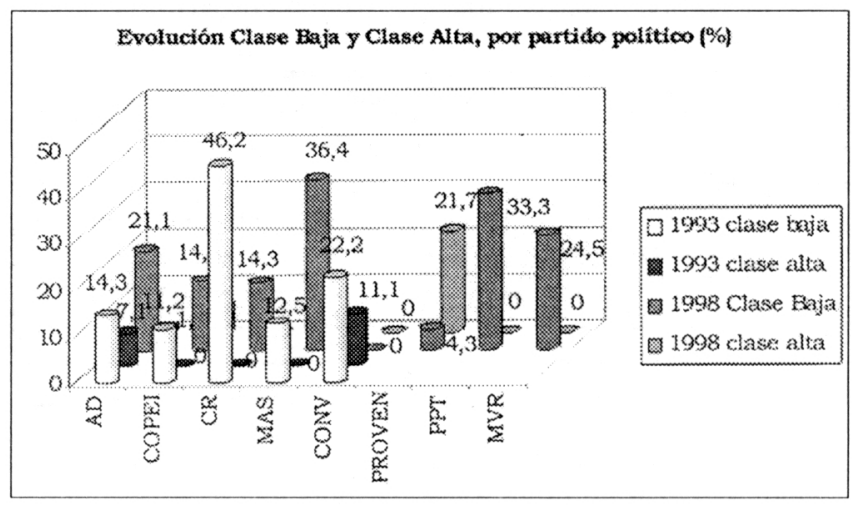

La distinción por partidos políticos tampoco agrega información significativa respecto a la diferenciación de clase social. En la Cámara de Diputados de 1993, señalaron pertenecer a la clase alta el $7 \%$ de los adecos y ningún copeyano, lo cual tampoco corroboraría la hipótesis de Von Beyme que señalaba que los miembros de los partidos de derecha o conservadores (COPEI) tendían a pertenecer a la clase alta. Con respecto a la izquierda los datos si corroborarían la hipótesis ya que el 78,6\% de los adecos, el 46,2\% de los diputados de Causa R y el $75 \%$ de los masistas señalaron pertenecer originalmente a la clase media-baja. En tanto, entre los diputados de los partidos no tradicionales que emergieron tras los comicios de 1998 se encuentran aquellos que se ubican en una clase social alta o media alta: el $27 \%$ de los diputados de Proyecto Venezuela se posicionó en ella, el $57 \%$ de los miembros de Convergencia indicó pertenecer a la clase media-alta, y el 18\% de los diputados de MVR se ubicó en la clase media-alta. En tanto, los diputados de los partidos tradicionales prefirieron señalar su pertenencia a la clase media ( $40,8 \%$ de adecos y $44,1 \%$ de copeyanos) y en menor medida a la baja.

\begin{tabular}{|c|c|c|c|c|c|c|c|c|c|c|c|c|c|c|c|c|}
\hline \multicolumn{17}{|c|}{ Cuadro II: Clase Social de Origen por Partido Político (\%) } \\
\hline & \multicolumn{2}{|c|}{$A D$} & \multicolumn{2}{|c|}{ COPEI } & \multicolumn{2}{|c|}{$\mathrm{CR}$} & \multicolumn{2}{|c|}{ MAS } & \multicolumn{2}{|c|}{ CONVEN } & \multicolumn{2}{|c|}{ PROVEN } & \multicolumn{2}{|c|}{ PPT } & \multicolumn{2}{|c|}{ MVR } \\
\hline & 1993 & 1998 & 1993 & 1998 & 1993 & 1998 & 1993 & 1998 & 1993 & 1998 & 1993 & 1998 & 1993 & 1998 & 1993 & 1998 \\
\hline Baja & 14.3 & 21.1 & 11.8 & 14.7 & 46.2 & $1+3$ & 12.5 & 36.4 & 22.2 & & & 4.3 & & 33.3 & & 24.5 \\
\hline Media-Baja & 78,6 & 31.6 & 64.7 & 23,5 & 46,2 & 28.6 & 75 & 22.7 & 4,4 & 14.3 & & 30,4 & & 25 & & 34 \\
\hline Media & $*$ & 40.8 & & H,1 & & 42,9 & & $\$ 0,9$ & & 28.6 & & 30,4 & & 16,7 & & 34 \\
\hline Media-Alla & . & 3.9 & 235 & 11.8 & 7.7 & $1+.3$ & 12.5 & & 22,2 & 57,1 & & 13 & & 25 & & 18,2 \\
\hline Alla & 7.1 & 1.3 & & 5.9 & 7.1 & & & & 11.1 & & & 27.1 & & & & \\
\hline n & 14 & 76 & 17 & 34 & 1.3 & 7 & 8 & 22 & 9 & 7 & & 23 & & 12 & & 53 \\
\hline
\end{tabular}


En cuanto al nivel de ingresos, la diferencia fundamental estriba entre los que solamente reciben ingresos como diputados y los que reciben ingresos adicionales, sean de otros trabajos o sean de rentas o beneficios empresariales. La mayor parte de los diputados venezolanos del período 1993-1998 entrevistados (40,5\%) afirma percibir entre 10.000 y 20.000 dólares norteamericanos anuales como ingresos totales, lo que al momento en el que se hizo la encuesta equivalía a una cantidad entre 170.000 y 340.000 bolívares. En tanto, casi el $25 \%$ de los entrevistados sostuvo que tenían un ingreso estimado entre 20.000 y 30.000 dólares anuales. Este dato mantiene la misma tendencia en la legislatura actual. El 56\% de los diputados entrevistados en este período afirma percibir una cantidad ubicada entre esas sumas y el $20 \%$ sostiene que se encuentra en la franja de los 20.000 a los 30.000 dólares anuales. Un dato a destacar es que los diputados que se dijeron percibir cifras mayores a los 50.000 dólares anuales se incrementó a más del 8\%.

\begin{tabular}{|c|c|c|}
\hline \multicolumn{3}{|c|}{ Cuadro III: Ingresos Anuales (en dólares norteamericanos) (\%) } \\
\hline & 1993 & 1998 \\
\hline (+) 50000 & 3,1 & 8,3 \\
\hline $40000-50000$ & 3,1 & 2,8 \\
\hline $30000-40000$ & 4,6 & 4,8 \\
\hline $20000-30000$ & 24,6 & 19,4 \\
\hline $10000-20000$ & 40,5 & 56 \\
\hline$(-) 10000$ & 12,3 & 6,3 \\
\hline
\end{tabular}

$\mathrm{Al}$ analizar el nivel de ingresos por partido político, los datos no presentan diferencias significativas ya que la mayor parte de los diputados, independientemente de su pertenencia partidaria, se ubicaron en la franja de los 10.00020.000 dólares anuales. Aún así caben destacar algunas diferencias. Por una parte, en la Cámara de 1993, un sector significativo de COPEI se ubicó entre los 20.000-30.000 dólares anuales así como también un sector de Convergencia. Por otra parte, en la Cámara de 1998, más del 20\% de los diputados de Proyecto Venezuela se ubicaron entre los 40.000 y 50.000 dólares y más del $15 \%$ de los legisladores de Convergencia y del PPT afirmaron recibir más de 50.000 dólares anuales.

\begin{tabular}{|c|c|c|c|c|c|c|c|c|c|c|c|c|c|c|c|c|}
\hline \multirow{3}{*}{\begin{tabular}{|l|} 
Cuadro IV: In \\
\end{tabular}} & \multirow{2}{*}{\multicolumn{2}{|c|}{ Al) }} & \multirow{2}{*}{\multicolumn{2}{|c|}{ COPEI }} & \multirow{2}{*}{\multicolumn{2}{|c|}{ CR }} & \multirow{2}{*}{\multicolumn{2}{|c|}{ MAS }} & \multirow{2}{*}{\multicolumn{2}{|c|}{ CONVEN }} & \multirow{2}{*}{\multicolumn{2}{|c|}{ PROVEN }} & \multirow{2}{*}{\multicolumn{2}{|c|}{ PPT }} & \multirow{2}{*}{\multicolumn{2}{|c|}{ MVR }} \\
\hline & & & & & & & & & & & & & & & & \\
\hline & 1993 & 1998 & 1993 & 1998 & 1993 & 1998 & 1993 & 1998 & 1993 & 1998 & 1993 & 1998 & 1993 & 1998 & \begin{tabular}{|l|}
1993 \\
\end{tabular} & 1998 \\
\hline 500000 & & 6.6 & 5.9 & 11.8 & 7.1 & 143 & & 9.1 & & 16.7 & & & & 16.7 & & 1.9 \\
\hline$\$ 10000.500000$ & 6.7 & 2.6 & & 2.9 & & & & 4.5 & 11.1 & & & 21.7 & & & & \\
\hline $30000-400000$ & & 6.6 & 5.9 & 2.9 & & $1+3$ & 12.5 & 4.5 & 11.1 & 16.7 & & 8.7 & & & & 1.9 \\
\hline $20000-300000$ & 26.7 & 14.5 & 47,1 & 32.4 & & 28.6 & 12.5 & 9.1 & 33,3 & 33,3 & & 34,8 & & 25 & & 17 \\
\hline $100000-200000$ & 40 & 60,5 & +1.2 & 47 & 38,5 & 42,9 & 50 & 72,8 & 33,3 & 33,3 & & 31,4 & & 50 & & 67,9 \\
\hline .100000 & 20 & 3.9 & & 2.9 & 23.1 & & 125 & & & & & & & 8.3 & & 11.3 \\
\hline $\mathrm{N}$ & 15 & 76 & 17 & 34 & 13 & 7 & 8 & 22 & 9 & 6 & & 23 & & 12 & & 53 \\
\hline
\end{tabular}

Si bien se encontraron algunas diferencias menores, se debe destacar que la mayor parte de los legisladores de ambas Cámaras se posicionaron en un nivel de ingresos de entre 10.000 y 20.000 dólares anuales. Aunque es una cantidad que está por encima de la media de ingresos en Venezuela, no se puede decir que sea una cantidad muy elevada y no permite hablar de aquel que lo percibe como perteneciente a una clase social alta. Esto se confirmaría con la opinión generalizada entre los diputados de 1993-1998 de que el salario que reciben es muy insuficiente para un $18.5 \%$, e insuficiente para un $63,1 \%$. Así, estas tendencias no parecen corroborar la hipótesis presentada en páginas anteriores acerca de que la mayoría de los legisladores pertece a la clase media-alta o alta, por lo menos en cuanto al nivel de ingresos. Este análisis añade una dificultad importante toda vez que no resulta apropiado medir estos datos sólo tomando en cuenta las opiniones de los diputados.

\section{- Datos Sociodemográficos}

\section{a. Educación}

La relación entre el alto nivel educativo y la pertenencia a la élite política parece ser una de las hipótesis más aceptadas y menos debatidas. Al respecto, Putnam señala que "...education is another important dimension of social stratification that is bighly correlated with political status..." (1976: 26)5. También Von Beyme señala que la educación “...era el factor más importante de distinción entre élites y no élites..." (1995:117). Sin embargo, él mismo sostiene que en el proceso de homogeneización social antes señalado, “...la formación universitaria es una condición suficiente pero no necesaria, para una carrera política..." (1976:118).

\section{Comparación Nivel de Postzrado en 1993 y 1998 por partido político $(\%)$}

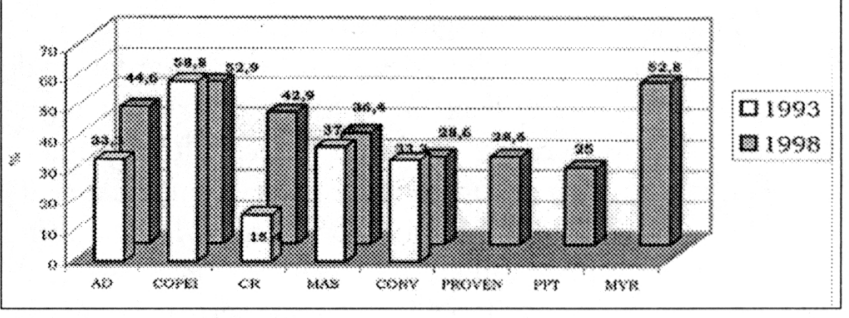

Por tanto, se trataría de corroborar hasta qué punto está generalizada la formación universitaria entre los legisladores venezolanos y en que medida constituye un ejemplo de homogeneización social en el sentido apuntado por Von Beyme. Cabe destacar que debido a la cada vez mayor homogeneización de la formación universitaria en este país, el elemento diferenciador se establecerá en términos de poseer estudios de postgrado. El mismo funcionará como un umbral mínimo, ya que con las características sociales de Venezuela, no se puede considerar un elemento demasiado elitista poseer estudios universitarios. En este sentido, disponer de estudios de postgrado sí marca realmente la diferencia.

En este sentido, y a la vista de los datos de la Investigación de Élites Parlamentarias en América Latina, tan sólo el $18,5 \%$ de los diputados no habían obtenido ningún título universitario. En el otro extremo, un 38,5\% ha hecho estudios de postgrado. En suma esto indica un nivel educativo moderadamente alto.

5. “... educación es otra dimensión importante de la estratificación social que está altamente correlacionada con el status político...". 
En la Cámara de 1993, el mayor nivel educativo lo poseían los diputados de COPEI, entre los cuales el 58,8\% había alcanzado a realizar estudios de postgrado. Los niveles más bajos de educación los tenían los diputados de Causa R y el MAS, de los que el 23,1 y el $25 \%$ respectivamente no manifestaron tener ningún título universitario. Con respecto a la Cámara en 1998, el mayor nivel educativo correspondía nuevamente a los diputados de COPEI $(52,9 \%$ ) (dato que mantiene la tendencia de la Cámara anterior), de Proyecto Venezuela $(68,2 \%)$ y del Movimiento Quinta República $(52,8 \%)$, integrante del Polo Patriótico. Pero al mismo tiempo los niveles más bajos de educación se encontraban en los mismos partidos, con la clara excepción de PROVEN donde todos los diputados habían alcanzado título universitario. En tanto, el 28,6 de los diputados de Causa R y el 16,7 de PPT no poseía ningún título universitario.

\begin{tabular}{|c|c|c|c|c|c|c|c|c|c|c|c|c|c|c|c|c|}
\hline \multicolumn{17}{|c|}{ Cuadro V: Nivel Educativo por Partido Político (\%) } \\
\hline & \multicolumn{2}{|c|}{$A D$} & \multicolumn{2}{|c|}{ COPEI } & \multicolumn{2}{|c|}{$C R$} & \multicolumn{2}{|c|}{ MAS } & \multicolumn{2}{|c|}{ CONVEN } & \multicolumn{2}{|c|}{ PROVEN } & \multicolumn{2}{|c|}{ PPT } & \multicolumn{2}{|c|}{ MVR } \\
\hline & 1993 & 1998 & 1993 & 1998 & 1993 & 1998 & 1993 & 1998 & 1993 & 1998 & 1993 & 1998 & 1993 & 1998 & 1993 & 1998 \\
\hline Primaria & 6.7 & 2.7 & 5.9 & & & & & & & & & & & & & 1.9 \\
\hline Sccundaria & 6.7 & 12.7 & 11.8 & 2.9 & 23.1 & 28,6 & 25 & 13.6 & 11.1 & & & & & 16.7 & & 9.4 \\
\hline Técnicos & & 1.1 & & 8.8 & & & & 18.2 & & 143 & & 14.3 & & 8.3 & & 7.5 \\
\hline Liniversitanios & 533 & 39.2 & 235 & 35.3 & 61.5 & 28.6 & 37.5 & 31.8 & 55,6 & 57,1 & & 57,1 & & 50 & & 28.3 \\
\hline Possterado & 333 & 44.6 & 58.8 & 52.9 & 15.4 & 42.9 & 37.5 & 36,4 & 33.3 & 28,6 & & 28.6 & & 25 & & 52.8 \\
\hline n & 15 & 74 & 17 & 34 & 13 & 7 & 8 & 22 & 9 & 7 & & 22 & & 12 & & 53 \\
\hline
\end{tabular}

No se podría sostener la hipótesis de Von Beyme referente a la homogeneización en cuanto al nivel educativo, sino que existen diferencias relativamente marcadas en cuanto al nivel educativo de los diferentes partidos. Lógicamente, no hay que desconocer que el nivel educativo está fuertemente relacionado con la clase social (Putnam, 1976), por lo que se redundaría en la identificación entre partidos de derecha, clase social alta y más alto nivel educativo y partidos considerados de izquierda, clase social más baja y menor nivel educativo.

\section{b. Edad, Género y Religión}

Existe una consideración generalizada de que los diputados son personas de edad media y que a medida que se avanza hacia la derecha en el espectro ideológico aumenta la edad. Lógicamente, la edad está fuertemente correlacionada con los años de dedicación a la política, por lo que de confirmarse esta hipótesis habría que decir que los diputados de partidos conservadores tienen una mayor trayectoria política. Así mismo, en general en la élite parlamentaria hay poca proporción de mujeres, menos aun en los partidos de derecha. Así lo señala Putnam (1976: 32) cuando indica que "...in statitical terms, women are the most underrepresented group in the political élites of the world..." ". En cuanto a religión la mayoría son católicos. Es decir, se trataría de ver hasta que punto existe en la actualidad el modelo clásico característico de los partidos de derecha (hombre, de edad media o avanzada y religioso) o si, por el contrario, se está reflejando en la composición de este grupo los cambios que aparentemente se está generando en la nueva derecha.

$\mathrm{Al}$ respecto se utiliza la variable edad para corroborar hasta qué punto esta hipótesis se confirma. Los datos indican que lo hace sólo parcialmente, ya que la edad media del

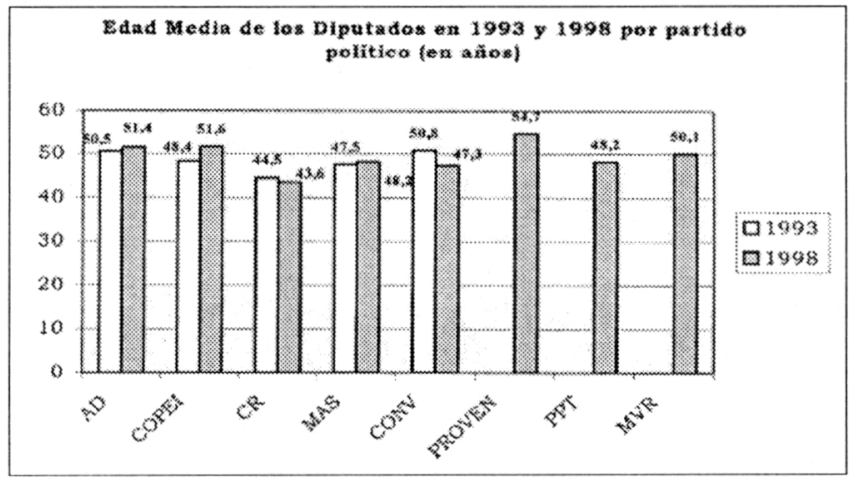

Congreso venezolano es de 50 años en el período 1993-1998 por lo que no existe una correlación fuerte entre partidos conservadores y mayor edad. Los diputados de COPEI en un alto porcentaje son personas de más de 55 años, aunque también hay un alto porcentaje de diputados menores de 45 años $(29,2 \%)$. Se podría decir que este partido tiene un alto porcentaje de diputados en el intervalo más alto de edad, consecuencia de su dilatada historia como organización partidista y además ha hecho un evidente esfuerzo por incorporar a personas jóvenes, lo que explicaría la polarización existente en cuanto a la edad dentro de este grupo parlamentario. Este proceso no se observa, sin embargo, en el caso de AD, donde el porcentaje de diputados menores de 45 años es sólo un 13,4\% frente al 53,9\% de Causa R o el 23,6 de COPEI. En cuanto a la Cámara de 1998, la edad media continúa siendo de 50 años y los diputados de Proyecto Venezuela $(54,7)$, COPEI $(51,6)$, y AD $(51,4)$ los de mayor edad; en tanto los diputados de Causa R $(43,6)$, MAS $(48,2)$, CONVEN $(47,3)$ y PPT $(48,2)$ presentan las medias de edad más bajas.

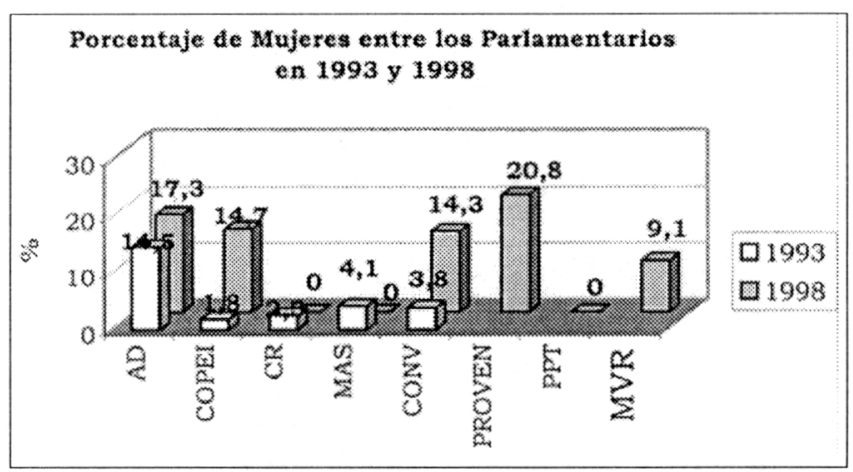

En cuanto al sexo de los diputados, las mujeres representaban únicamente un 6\% de la Cámara de Diputados del período 1993-1998. Por partidos, es AD el que presenta un mayor número de diputadas, exactamente un $14,5 \%$. Sin embargo, el resto de formaciones política solo tienen una representante del sexo femenino entre su grupo, lo que significa un $1,8 \%$ en el caso de COPEI, un $4,1 \%$ en el del MAS, un 2,5\% en el de Causa $\mathrm{R}$ y un 3,8\% en el de Convergencia. Por tanto, en la Cámara de 1993, se puede decir que con la excepción de $\mathrm{AD}$, los partidos políticos

6. “... en términos estadísticos, las mujeres son el grupo más subrepresentado en las élites políticas del mundo...”. 
venezolanos no habían hecho un esfuerzo de incorporación de mujeres al recinto parlamentario, estando en unos niveles extremadamente bajos la proporción de mujeres. Esta situación se ve modificada para la Cámara de 1998. La participación de las mujeres en la misma se ha incrementado a un $11,5 \%$. De los 207 legisladores, 30 corresponden al sexo femenino. Los partidos que más fomentan la participación de la mujer entre sus filas son Proyecto Venezuela, donde la presencia femenina alcanza el $20,8 \%$ de sus representantes, y AD donde el $17,3 \%$ de sus legisladores son mujeres. Cabe destacar que para la última elección se incluyó una norma electoral para incentivar la presencia de la mujer en el Congreso. Esa norma establecía que el 30\% de los candidatos de lista deberían ser cuota femenina.

\begin{tabular}{|c|c|c|c|c|c|c|c|c|c|c|c|c|c|c|c|c|}
\hline \multicolumn{17}{|c|}{ Cuadro VI: Religión de los Diputados según Partido Político (1993) (\%) } \\
\hline & \multicolumn{2}{|c|}{$\mathrm{AD}$} & \multicolumn{2}{|c|}{\begin{tabular}{|c|} 
COPEI \\
\end{tabular}} & \multicolumn{2}{|c|}{$C R$} & \multicolumn{2}{|c|}{ MAS } & \multicolumn{2}{|c|}{ CONVEN } & \multicolumn{2}{|c|}{ PROVEN } & \multicolumn{2}{|c|}{ PPT } & \multicolumn{2}{|c|}{ MVR } \\
\hline & 1993 & 1998 & 1993 & 1998 & 1993 & 1998 & 1993 & 1998 & 1993 & 1998 & 1993 & 1998 & 1993 & 1998 & 1993 & 1998 \\
\hline Católicos & 9 & 93,5 & 100 & 94.1 & 75 & 100 & 89 & 77,3 & 57 & 85.7 & & 82.6 & & 66,7 & & 71,7 \\
\hline Oras & 6 & 93 & & & & & 14 & 4.5 & & 14,3 & & 14 & & 8.3 & & 13,2 \\
\hline Atcos & & 1,3 & & 2.9 & 25 & & $x$ & 18,2 & II & & & 4,3 & & 2.5 & & 15,1 \\
\hline$n$ & 17 & $\pi$ & 18 & 34 & 12 & 1 & 7 & 22 & 9 & 7 & & 23 & & 12 & & 53 \\
\hline
\end{tabular}

En cuanto a la religión, el $80 \%$ de los diputados entrevistados en la Cámara de 1993 declararon ser católicos. Por partidos, este porcentaje se eleva hasta el 100\% en el caso de COPEI y desciende hasta el 69,2\% en el caso de Causa R o al $50 \%$ en el del MAS. Sin embargo, consultados sobre su asistencia a los servicios religiosos, solo un $16,9 \%$ dice acudir una vez por semana. La mayoría señala que acude irregularmente (41,5\%). Los datos para la Cámara de 1998 no se manifiestan diferentes a los de la legislatura anterior. Entre los diputados, la mayoría también se define como católicos $(81 \%)$. Entre los diputados de los partidos no tradicionales se da la representación de nuevas religiones en el Congreso. Grupos de legisladores de Convergencia $(14,3 \%)$, de Proyecto Venezuela $(14,0 \%)$ y de MVR $(13,2 \%)$ pertenecen a otras religiones y son los diputados de MAS $(18,2)$ y MVR $(15,1)$ los que en mayor número señalan ser ateos. En cuanto a la práctica religiosa, nuevamente son los diputados de COPEI los que en mayor proporción acuden regularmente a los servicios religiosos $(23,5 \%$ acuden al menos una vez por semana). Por tanto, se confirma el modelo de diputado perteneciente a un partido de derecha: hombre, edad mediana-avanzada y religioso, aunque en el caso de COPEI existe un grupo de edad joven que está transformando esta tendencia. Los datos de ambas Cámaras permiten sostener que en cuanto a la religión el Congreso actúa en espejo de la sociedad venezolana, dado que la amplia representación en las mismas de esta religión coincide con su extensión entre sus ciudadanos.

\section{- Antecedentes Familiares en la política ${ }^{7}$}

Poseer antecedentes familiares de dedicación a la política es un factor explicativo de la dedicación del diputado y, por consiguiente, de su pertenencia a la élite política. Putnam (1976:57) señala al respecto que el linaje, al igual que otros factores adscriptos a la persona explica, en gran medida, la pertenencia a la élite, en detrimento de los factores adquiridos. Indica que los lazos familiares juegan un papel significativo en el reclutamiento político incluso en los sistemas no hereditarios. Pone como ejemplo el caso francés de la III ${ }^{\text {ra }}$ República (1870-1940) en el que un séptimo de los diputados tenían relaciones de parentesco entre sí, o el de Estados Unidos, en el que un décimo de los diputados entre 1790 y 1960 tenían parientes que también habían sido legisladores. Por tanto, según esta hipótesis, un porcentaje alto de diputados pertenecería a una familia con algún antecedente de dedicación a la política.

Para corroborar esta hipótesis se utiliza la información proporcionada por las preguntas de si tenían familiares dedicados a la política, qué grado de parentesco les unía y si militaban en el mismo partido. Evidentemente, ya es significativo contar con cualquier tipo de familiar dedicado a la política, aunque no es lo mismo tener un ascendente que un descendiente para el significado de esta hipótesis. Tampoco es lo mismo tener un familiar directo y que, además, esté afiliado al mismo partido, que un familiar lejano perteneciente a un partido distinto.

A la vista de los datos de la Investigación de Élites Parlamentarias en América Latina, y en referencia a los familiares en general de los diputados del período 1993, en Venezuela llega hasta el $57,4 \%$ el porcentaje de diputados encuestados que tienen o han tenido algún familiar dedicado a la política. Por partidos son los diputados del MAS los que en mayor proporción dijeron tener algún político en la familia (75\%), frente al 61,5\% de Causa R, al 62,5\% de COPEI y al 46,7 de AD. Teniendo en cuenta estos datos, los diputados de los partidos tradicionales (AD y COPEI) no son los que cuentan con lazos de parentesco dentro de la política. Comparados con los datos que ofrece Putnam, se observa que el factor del linaje familiar en el caso venezolano es fuertemente explicativo de la pertenencia a la élite parlamentaria.

\begin{tabular}{|c|c|c|c|c|c|c|c|}
\hline \multicolumn{6}{|c|}{ Cuadro VII: Existencia de familiares dedicados a la política según partido político (1993) (\%) } \\
\hline & $\mathrm{AD}$ & $\mathrm{COPEI}$ & $\mathrm{CR}$ & $\mathrm{MAS}$ & CONVEN & OTROS & TOTAL \\
\hline SI & 46,7 & 62,5 & 61,5 & 75,0 & 44,4 & 50,0 & 57,1 \\
\hline NO & 53,3 & 37,5 & 38,5 & 25,0 & 55,6 & 50,0 & 42,9 \\
\hline Total & 15 & 16 & 13 & 8 & 9 & 2 & 63 \\
\hline
\end{tabular}

Fuente: Investigación Elites Parlamentarias en América Latina

Sin embargo, las tendencias empiezan a variar al desglosar este dato. No es lo mismo tener un único familiar que tener varios. Se podría afirmar que tener más de tres es pertenecer a una familia de tradición política. En este sentido, son los diputados del MAS y COPEI los que tienen un mayor número de familiares dedicados a la política, con $37,5 \%$ y $29,4 \%$ respectivamente de diputados que tienen más de 3 familiares en estas condiciones. Llama la atención la diferencia de Convergencia con el resto de los partidos, de los que "sólo" el 44,4\% tiene algún familiar en la política, frente al $15,6 \%$ que no tiene ninguno.

En cuanto al parentesco que une a los diputados con sus familiares políticos, son de los partidos tradicionales los que

7. Este apartado está dedicado únicamente al análisis de los datos de la legislatura de 1993 por carecer de la información del Congreso actual. 
dicen tener mayor porcentaje de familiares directos, ascendentes (padre o madre), dedicados a la política ( $50 \%$ de los diputados en el caso de COPEI y $57,1 \%$ en el caso de AD, frente a un 37,5\% de diputados de Causa R, un 33,3\% de los diputados encuestados del MAS o un $25 \%$ de los de Convergencia). En resumen, aunque los diputados del MAS y de Causa $\mathrm{R}$ son los que en mayor proporción declaran tener algún familiar dedicado a la política, los datos permiten pensar que los diputados de COPEI y AD tienen una mayor proporción de pertenencia a una familia de tradición políti$\mathrm{ca}$, teniendo en cuenta la variable del número de familiares y del parentesco que les une.

\begin{tabular}{|c|c|c|c|c|c|c|c|}
\hline \multicolumn{8}{|c|}{$\begin{array}{l}\text { Cuadro VIII: Tipo de parentesco entre el familiar dedicado a la política y el diputado según partido } \\
\text { político (1993) (\%) }\end{array}$} \\
\hline & $\mathrm{AD}$ & COPEI & $\mathrm{CR}$ & MAS & CONVEN & OTROS & TOTAL \\
\hline Abuclo & & & & 16.7 & & & 2.8 \\
\hline Padre/Madrc & 57.1 & 50,0 & 37.5 & 33.3 & 25.0 & & 41.7 \\
\hline Hermano/a & 28.6 & 20.0 & 25.0 & 16.7 & 25.0 & & 22.2 \\
\hline Otro familiar & 14.3 & 30.0 & 37.5 & 33.3 & 50.0 & $100)$ & 33.3 \\
\hline Total & 7 & 10 & 8 & 6 & 4 & 1 & 3.6 \\
\hline
\end{tabular}

Otro dato interesante tiene que ver con la trayectoria familiar del diputado, es decir, el partido político al que pertenece o perteneció. En general, se puede decir que esta hipótesis del linaje, como determinante de la incorporación de la élite parlamentaria, es más fuerte si el diputado continúa también la tradición partidista o de partidos afines, puesto que a los factores de socialización que implica tener familiares dedicados a la política, se añade uno de los elementos que según Putnam (1976: 49) tiene la mayor fuerza explicativa para el reclutamiento de las élites, la del partido político. En este caso, en Venezuela se puede decir que, en general, los diputados continúan tradiciones familiares de adscripción partidista, aunque hay grandes diferencias, como por otra parte es lógico, entre los partidos tradicionales y los partidos que surgieron en la arena política en aquel momento (Causa R y Convergencia). El 100\% de los familiares de diputados de COPEI con antecedentes familiares de dedicación a la política pertenecieron o pertenecen a COPEI; en el caso de AD este porcentaje llega al $66,7 \%$, aunque hay también casos de familiares pertenecientes al MAS y al PCV. El 50\% de los familiares de Causa $\mathrm{R}$ pertenecen o pertenecieron a $\mathrm{AD}$ y el resto se reparte entre el PCV y la guerrilla; del MAS, los familiares pertenecen o pertenecieron al PCV $(50 \%)$ o a $\mathrm{AD}(33,3)$. Los familiares de Convergencia pertenecieron en su mayoría a COPEI (75\%).

Por tanto, la hipótesis planteada por Putnam se confirma totalmente en el caso venezolano. Además la influencia de los antecedentes familiares de dedicación a la política es mayor en el partido conservador, COPEI, donde hay que

\begin{tabular}{|c|c|c|c|c|c|c|c|}
\hline \multicolumn{7}{|c|}{ Cuadro IX: Partido Político del Familiar según Partido Político del diputado (1993) (\%) } \\
\hline & AD & COPEI & CR & MAS & CONVEN & OTROS & TOTAL \\
\hline AD & 66,7 & & & & & & 30,3 \\
\hline COPEI & & 100 & & & 75,0 & & 33,3 \\
\hline CR & & & 50,0 & 33,3 & & & 3,0 \\
\hline MAS & 16,7 & & & & & 100 & 6,1 \\
\hline CONVE. & & & & & 25,0 & & 3,0 \\
\hline PCV & 16,7 & & 25,0 & 50,0 & & & 18,2 \\
\hline GUERR. & & & 12,5 & & & & 3,0 \\
\hline OTROS & & & & 16,7 & & & 3,0 \\
\hline Total & 7 & 10 & 8 & 6 & 4 & 1 & 36 \\
\hline Fucnte: Investigación Elites Parlamentanias en América Latina & & & \\
\hline
\end{tabular}

considerar la mayor tradición familiar de pertenencia a un mismo partido. Este último dato puede ser también aplicado a $\mathrm{AD}$. En resumen, se puede decir que los diputados venezolanos siguen las tradiciones familiares de adscripción ideológica, toda vez que continúan la tradición de pertenecer al mismo partido en el caso de COPEI, partido que ha sido el único representante de la ideología conservadora y democristiana hasta el año 1993, o en la misma línea ideológica en el resto de los partidos, a tenor de los datos ofrecidos por los diputados de AD, del MAS y Causa R, según los cuales no han tenido ni tienen familiares pertenecientes a partidos de derecha.

La existencia o no de antecedentes familiares determina en gran medida la trayectoria política del diputado, en el sentido de que pertenecer a una familia de tradición política ligada a un único partido se correlaciona positivamente con la trayectoria del diputado también ligado a un único partido. Teniendo en cuenta los datos apuntados anteriormente, serían los diputados de COPEI, y en menor medida los de $\mathrm{AD}$, los que habrían militado en un número menor de partidos.

En este caso, se les preguntó a los diputados en qué partido político habían militado y, posteriormente, se cruzó esta variable con la del partido político familiar. La mayor movilidad se produce entre diputados de Causa R y el MAS. Los diputados de los partidos tradicionales son los que menos movilidad presentan. El 100\% de los diputados de AD solo ha militado en $\mathrm{AD}$, así como el $82,4 \%$ de los diputados de COPEI. En cambio, entre los diputados del MAS, el 25\% procede de AD o COPEI y el $37,5 \%$ del PCV. Entre los de Causa R, tan solo el 30,8\% ha militado únicamente en Causa R. Un 38\% ha militado en el PCV y un $23,1 \%$ en otros partidos menores. Entre los de Convergencia, un $77,8 \%$ procede de COPEI.

Cruzando esta variable con la del partido político del familiar, se observa que la tendencia se confirma y que los partidos tradicionales siguen esta pauta. El 76\% de los diputados que han militado en COPEI tienen algún familiar que también ha militado en COPEI. Este porcentaje desciende solo al 66,7\% en el caso de AD. En el resto de los diputados que han militado en partidos distintos, no se confirma esta tendencia. Nuevamente, hay que tener en cuenta la reciente creación de algunos de ellos y la dinámica del sistema de partidos venezolano de los últimos treinta años, en el que los partidos que han controlado los resortes del poder han sido AD y COPEI, lo que explicaría la mayor continuidad dentro de esas familias partidarias.

\section{- Profesionalización}

Una hipótesis básica establece que la renovación política de los grupos parlamentarios es mayor entre los nuevos partidos que entre los partidos tradicionales (AD y COPEI), entre los que además, la media de dedicación a la política es mayor. Sin embargo, este dato obvio tiene importantes consecuencias de cara a la profesionalización de la política y los resultados que de ello se derivan. Por político profesional se entiende, de acuerdo con Sartori (1992: 177), a la persona que se ocupa de manera estable de la política. No serían, por 
tanto, políticos profesionales aquellos que se dedican de forma ocasional o durante un período de tiempo limitado a la vida política y que poseen una profesión que continúan ejerciendo incluso cuando entran a formar parte del Parlamento. La profesionalización, que en principio puede percibirse como una condición positiva, en tanto las ventajas que se derivan de contar con políticos expertos y que conocen bien el oficio, es percibida también como un elemento negativo, propia de personas que no tienen ningún oficio previo. Von Beyme (1995:122) señala que la "...la profesionalización de los políticos ha reconocido tempranamente como vehículo para la constitución de una especie de clase política, ya que de este proceso conduce a un necesario extrañamiento del político con respecto a su profesión de origen..." Por tanto, se trataría de ver hasta qué punto la élite parlamentaria venezolana se ha profesionalizado, comprobando si se confirma la hipótesis de Von Beyme (1995:124), según la cual, en la actualidad, "... los políticos tienen una percepción de su papel más intensamente profesionalizado que las élites anteriores (...) En la nueva democracia de partidos no sólo se eligen campos de especialización acordes con la formación, sino que los políticos intentan incluso adquirir un papel acorde con el contenido del cargo...".

Siguiendo a Von Beyme (1995), las variables explicativas del grado de profesionalización de la política serían el número de años en ejercicio, la actividad adicional que desempeña y la disposición a regresar a esta actividad en caso de que no la simultanee. Para el caso venezolano vamos a utilizar las dos primeras ${ }^{8}$. En cuanto a los años en ejercicio, se emplea tanto la variable de los años en ejercicio como diputado como los años de dedicación a la política. Utilizando la información que cada diputado aportó con relación al año en que fue elegido por primera vez diputado, se pueden sacar conclusiones en cuanto a la renovación de la élite parlamentaria y la relación existente por partido político.

En general, se puede decir que la legislatura de 1993 puede denominarse como un Congreso nuevo, ya que el $43,1 \%$ de los diputados ha sido elegido por primera vez en ese año. La media de entrada en la Cámara de Diputados es 1985, es decir, entre dos y tres legislaturas anteriores. Lógicamente, los resultados en los casos de Causa $\mathrm{R}$ y Convergencia no pueden ser comparados con el resto de partidos, ya que son partidos que prácticamente se estrenan en la Cámara de Diputados. La comparación se puede establecer entre los otros tres partidos importantes: AD, COPEI y el MAS. La renovación ha sido mucho mayor en el caso de COPEI, en el que el 41,2\% de sus diputados han sido elegidos como tales en 1993, frente a un 13,3\% de adecos (lo que se relacionaría con la estructura de edades antes señalada). Un 40\% de los diputados de AD fueron elegidos por primera vez en 1983, es decir, que llevan ya tres legislaturas y un $46,6 \%$ fueron elegidos con anterioridad.

En cuanto a los años de dedicación a la política, la media es de 30 años, aunque hay una gran dispersión (10,8 años de desviación standard), ya que el rango es de 47 años (desde 50 años de dedicación a 3 años). En general se puede decir que el parlamento venezolano tiene una amplia trayectoria política, ya que el $31,7 \%$ se dedica a la política desde hace más de 40 años, mientras que sólo el 7,7\% se dedica desde hace 15 años o menos. Es significativa la comparación entre la media de dedicación a la política y la de comienzo como diputado (entre 2 y 3 legislaturas, es decir, entre 10 y 15 años). De ello se deduce que el diputado pasa entre 15 y 20 años trabajando en política antes de ser elegido.

Por partidos, son los diputados de Convergencia y del MAS entre los que hay mayor proporción que se dedican a la política desde hace más de 35 años $(55,5 \%$ y $37,5 \%$ respectivamente). No hay grandes diferencias en ese tramo con respecto a los diputados de COPEI y $\mathrm{AD}(35,5 \%$ y $33,4 \%$ respectivamente), aunque sí en relación con los diputados de Causa R, entre los que solo un $7,7 \%$ se dedica a la política desde hace más de 35 años, es decir, que pertenecen a la generación del 58. En el otro extremo, ningún diputado de AD, MAS o Convergencia actúa en política desde hace 15 años o menos, frente a un $11,8 \%$ de COPEI y un 30,8\% de diputados de Causa R. Por tanto, de estos datos se pueden inferir que son los de Causa $\mathrm{R}$ los que menor experiencia política tienen y que el resto de los partidos cuentan con legisladores de amplia trayectoria política, destacando el grupo de reciente incorporación dentro de COPEI.

\begin{tabular}{|c|c|c|c|c|c|c|c|}
\hline \multicolumn{8}{|c|}{ Cuadro X: Año de elección por primera vez como diputado según partido político (1993) (\% } \\
\hline & $\mathrm{AD}$ & COPEI & $\mathrm{CR}$ & MAS & CONVEN & OTROS & TOTAL \\
\hline 1958 & & 5,9 & & & 11,1 & & 3,0 \\
\hline \multicolumn{8}{|l|}{1963} \\
\hline 1968 & 13.3 & 5,9 & & & 11,1 & & 6,2 \\
\hline 1973 & 13,3 & & & & & & 3,1 \\
\hline 1978 & 20,0 & 5,9 & & & & & 6.2 \\
\hline 1983 & 40,0 & 23.5 & 7,7 & 62.5 & 11.1 & 33,3 & 27,7 \\
\hline 1988 & & 17.7 & 7,7 & 12,5 & 11,1 & 33,3 & 10,8 \\
\hline 1993 & 13,3 & 41,2 & 84,6 & 25,0 & 55,6 & 33,3 & 43,1 \\
\hline Total & 15 & 17 & 13 & 8 & 9 & 3 & 65 \\
\hline
\end{tabular}

Esta diferenciación por partidos continúa con la que establece la variable del año de elección por primera vez como diputados. Lógicamente, el 84,6\% de los legisladores de Causa $\mathrm{R}$ fueron elegidos por primera vez en 1993. Este porcentaje desciende al 55,6\% en el caso del otro partido de reciente incorporación a esta Cámara, Convergencia, de lo que se infiere que previamente fueron diputados de COPEI. Además, este trasvase de representantes de COPEI a Convergencia puede explicar el mayor número de nuevas incorporaciones en el tradicional partido democristiano $(41,2 \%$ de diputados fueron elegidos por primera vez como tales en 1993, frente al $13,3 \%$ de adecos o el $25 \%$ de masistas).

En cuanto a los indicadores que utiliza Von Beyme para analizar el caso alemán, el $60 \%$ de los diputados tiene otra ocupación aparte de la política. Este porcentaje llega al $93,3 \%$ en el caso de los adecos o al $75 \%$ en el de los masistas. Por el contrario, son los partidos de más reciente incorporación entre los que existe una mayor proporción de dedicación exclusiva a la política $(46,2 \%$ en el caso de Causa $\mathrm{R}$ y el 100\% de los diputados de Convergencia).

La profesión previa a la dedicación a la política es mayoritariamente cualquier tipo de profesión liberal $(45,8)$, incluidos los abogados. Le siguen en importancia los profesores $(18,8 \%)$ de los que el $7,8 \%$ son profesores universitarios y el trabajo dentro del partido político o como sindicalista

8. Se medirán los dos indicadores en la Legislatura de 1993, dado que no se cuenta con datos de los años dedicados a la política para la Legislatura de 1998. A modo complementario se presentarán los datos de las profesiones de los diputados de la Legislatura de 1998. 
$(6,3 \%)$. Es significativo que el $20,3 \%$ de los diputados indicaran que no tenían ninguna otra profesión previa a su dedicación como político/diputado. Otras profesiones que están representadas dentro de la Cámara son: pequeño empresario $(3,2 \%)$, trabajadores industriales $(3,1 \%)$ y funcionarios de alto nivel $(1,6 \%)$.

Por partidos, el mayor porcentaje de trabajadores de partidos o sindicalistas da entre los diputados de AD $(13,3 \%)$, seguido de los de Causa $\mathrm{R}(7,7 \%)$ aunque en este caso el porcentaje es bastante bajo tratándose de un partido de fuerte componente sindicalista. Entre los diputados que no tenían ninguna otra ocupación, la proporción más alta se da entre los masistas $(50 \%)$ y copeyanos $(25 \%)$ y el menor de los adecos $(6,7 \%)$ y de Causa R (15,4\%). Entre los diputados de Convergencia, la mayoría se dedica a profesiones liberales $(55,6 \%)$, seguido de profesores $(22,2 \%)$. Por su parte, los profesores son en su mayoría diputados de $\mathrm{AD}$, de COPEI y de Convergencia y dentro de éstos, el $40 \%$ de los profesores universitarios pertenecen a COPEI. Los trabajadores industriales pertenecen en su totalidad a Causa R. En suma, según este criterio, se observa una gran homogeneidad social en cuanto a los diferentes partidos políticos, aunque, de acuerdo con lo planteado por Von Beyme (1995:108) se confirma también para el caso venezolano una tendencia a la heterogeneización de las profesiones.

Entre los diputados elegidos en noviembre de 1998 la tendencia se mantiene, puesto que la mayoría de los legisladores cuenta con una profesión liberal (AD, 63,9\%; COPEI, 64,7\%; CR, 57,2\%; MAS, 27,3\%; PROVEN, 60,9\%, PPT, 41,7 y MVR, $31,4 \%$ ). Aquellos diputados que se han dedicado únicamente a la carrera política pertenecen mayoritariamente a Patria para Todos $(16,7 \%)$. En tanto, entre los diputados que se han desempeñado como profesores encontramos una proporción importante entre los copeyanos $(17,6 \%)$, los integrantes del Movimiento Quinta República $(11,8 \%)$ y los de Causa R $(14,3 \%)$. Estos datos indican en parte los magros niveles de dedicación exclusiva a la política entre los diputados venezolanos puesto que la mayoría de los legisladores ha señalado su desarrollo profesional paralelo a la vida política.

\begin{tabular}{|c|c|c|c|c|c|c|c|c|c|c|c|c|c|c|c|c|}
\hline & \multicolumn{2}{|c|}{$\mathrm{AD}$} & \multicolumn{2}{|c|}{ COPEI } & \multicolumn{2}{|c|}{ CR } & \multicolumn{2}{|c|}{ MAS } & \multicolumn{2}{|c|}{ CONVEN } & \multicolumn{2}{|c|}{ PROVEN } & \multicolumn{2}{|c|}{ PPT } & \multicolumn{2}{|c|}{ MVR } \\
\hline & 1993 & 1998 & 1993 & 1998 & 1993 & 1998 & 1993 & 1998 & 1993 & 1998 & 1993 & 1998 & \begin{tabular}{|l|}
1993 \\
\end{tabular} & 1998 & \begin{tabular}{|l|}
1993 \\
\end{tabular} & 1998 \\
\hline $\begin{array}{l}\text { Dirigente } \\
\text { Político }\end{array}$ & 13,3 & 4,1 & 6,3 & 2,9 & 7,7 & & & 4.5 & & & & & & 16,7 & & 3,9 \\
\hline \begin{tabular}{|l} 
Profesión \\
Liberal \\
\end{tabular} & 46,7 & 63,9 & 37,5 & 64,7 & 46,2 & 57,2 & 9) & 27,3 & 55,6 & 57,2 & & 60,9 & & 41,7 & & 31,4 \\
\hline Tícnicos & & 1.1 & & & & 14.3 & & 9.1 & & & & & & & & \\
\hline Proficon & 26.7 & 8.2 & $\underline{x}$ & 17.6 & 7.7 & 14.3 & & & & & & & & & & 11.8 \\
\hline Otras & 31.3 & & 6.7 & & 23.1 & & 8) & & 22.2 & & & 8.6 & & & & \\
\hline $\mathrm{n}$ & 15 & 74 & 16 & 34 & 13 & 7 & 8 & 2 & 9 & 7 & & 23 & & 12 & & 51 \\
\hline
\end{tabular}

Por último, el 74,4\% de los diputados de la Legislatura de 1993 consideró que su profesión les daría más dinero que la política, aunque este porcentaje varía de unos partidos a otros, estimando mayor esta diferencia los diputados de COPEI y el MAS. Así se explica que mayoritariamente consideren que la política supone un enorme sacrificio $(70 \%$ está muy de acuerdo con esa afirmación) y que “...dedicándose a la política no se aspira a más beneficio que el reconocimiento y la satisfacción por el trabajo bien hecho..."
(76,9\% está de acuerdo o muy de acuerdo), en detrimento de motivaciones como el enriquecimiento personal (el 67,7\% está en completo desacuerdo) y, en menor medida, el ascenso social $(41,6 \%$ está en desacuerdo o en completo desacuerdo) o la consideración de la política como una profesión cualquiera $(36,9 \%$ está en desacuerdo o en completo desacuerdo). Es decir, que los diputados de la legislatura anterior consideraron la política como algo excepcional que les supone un sacrificio personal y económico pero a la que sin embargo se dedican a lo largo de gran parte de su trayectoria vital. No se podría hablar por tanto de una profesionalización, sino, en todo caso de políticos semiprofesionales, que no son ocasionales pero que no perciben que la política sea su profesión, ya que mayoritariamente la compaginan con otra. Sin embargo, hay que establecer importantes diferencias entre partidos, ya que los partidos políticos que cuentan con legisladores con amplia experiencia política, son los que en mayor proporción compaginan el cargo de diputado con su otra profesión. Por el contrario, los representantes de partidos de reciente incorporación al Congreso son los más proclives a dedicarse únicamente al cargo de diputado. Por tanto, hay que concluir que la Cámara de Diputados de 1993 estaba compuesta por políticos semiprofesionales, pues si bien se dedicaban a la política desde hace años, mantenían su profesión anterior.

\section{CONCLUSIONES}

El estudio de las características sociodemográficas de las élites parlamentarias venezolanas señala que se ha dado una renovación importante entre los legisladores de las dos últimas legislaturas (1993 y 1998) y que en los últimos diez años se ha generado un proceso de transformación significativo que ha llevado a reflejar al interior del Congreso los cambios existentes en la sociedad. Venezuela ha dejado de ser en los últimos años un sistema bipartidista, caracterizado por el predominio por cerca de treinta años de dos grandes partidos tradicionales (COPEI y AD) para transformarse primero en un sistema multipartidista limitado y luego en uno extremo.

Los cambios experimentados en el sistema de partidos pueden ser interpretados desde distintos enfoques. Uno de ellos sería el de pensarlos como resultados de las transformaciones en las preferencias de los votantes, que buscan con su voto alternativas distintas para superar los críticos y cotidianos problemas sociales y económicos de su país. En 1993 la opción fue elegir partidos políticos que ofrecían cambios parciales pero que se mantenían dentro del esquema político-partidario. El crecimiento de Causa R y de Convergencia pueden explicarse desde este punto de vista. Cinco años después el cambio se busca en otras opciones, más radicales que las anteriores, y con un discurso netamente antipartidista. El Movimiento Quinta República, Patria para Todos y Proyecto Venezuela, más allá de sus diferencias, representan alternativas a los partidos tradicionales y una nueva manera de "bacer política", en particular, el grupo político encabezado por Chávez.

En este contexto los datos encontrados en el análisis sociodemográfico indican algunas transformaciones en las 
élites parlamentarias venezolanas. Los mismos muestran que el Congreso de 1993 es un Congreso Nuevo, bastante heterogéneo, que no puede entenderse a la élite política como un cuerpo uniforme y que en los últimos diez años se ha reflejado cada vez más la pluralidad de la sociedad venezolana.

En términos generales, los legisladores resultaron ser hombres, católicos; de edad media (50 años); con altos niveles de formación, principalmente en profesiones liberales y, en una proporción significativa, con especializaciones de postgrado. Además, los legisladores de 1993 tenían familiares dedicados a la vida política por lo que esta condición parecería ser una variable explicativa de su pertenencia a la élite, por lo menos desde la perspectiva presentada por Putnam (1976). De este modo se podría decir que los diputados continúan tradiciones familiares de adscripción partidista, aunque hay grandes diferencias, como por otra parte es lógico, entre los partidos tradicionales y los partidos que surgieron en la arena política en 1993 (Causa R y Convergencia). El presente estudio indica que respecto a la relación entre posición socioeconómica y pertenencia a la élite política, en el Congreso venezolano no se corroboraría la hipótesis del modelo de aglutinación presentado por Putnam (1976), sino que abonaría lo sostenido en el modelo de la independencia acerca de la inexistencia de relación entre posición socioeconómica y élite política. Por tanto este dato indica dos cuestiones. Por una parte la pertenencia a esa élite no está sujeta al nivel de ingresos, ni a la clase social de origen del legislador sino a otras cuestiones y, por otra parte, esto también muestra el carácter heterogéneo de los miembros del Congreso Venezolano.

Finalmente, la composición del Congreso refleja en la actualidad las transformaciones del sistema político venezolano, en tanto no sólo se ha producido cambios a nivel de los social, sino sobre todo, ha habido cambios en cuanto a la vinculación de la sociedad con las instituciones políticas. El Congreso ya no es una institución ocupada por la élite tradicional partidista, sino que constituye una muestra de la incorporación de nuevos actores y grupos a la institucionalidad política.

\section{BIBLIOGRAFÍA}

AlCÁntara, M. y Llamazares V., I. (1997): “El análisis de los diputados latinoamericanos en el contexto de los estudios sobre la clase política. Características, objetivos y estrategias de investigación”, América Latina Hoy, 16: 15-28.

Close, David (1995): Legislatures and the New Democracies in Latin America. Boulder: Lynne Rienner Pub.

Diamond, L. (1993): Political Culture and Democracy in Developing Countries. Boulder: Lynne Rienner Pub.

Higley, J. y Gunther, R. (1992): Elites and Democratic Consolidation in Latin America and Southern Europe, Cambridge University Press.

Linz, J. J. y STEPAN, A. (1997): “Toward Consolidated Democracies” in Diamond, L., Plattner, M., Yun Han Chu y Hung-Mao Tien (Edit.) Consolidating The Third Wave Democracies. Baltimore y London: The Johns Jopquins University Press.

Putnam, RoBerT (1976) The Comparative Study of Political Elites. Englewood Cliffs, New Jersey: Prentice Hall.

SARTORI, G. (1976): Parties and Party Systems: a Framework. for Political Analysis. Cambridge: Cambridge University Press.

- (1992): Elementos de Teoría Política. Madrid: Alianza

Von Beyme, K. (1995): La Clase Política en el Estado de Partidos. Madrid: Alianza Universidad.

\section{RESUMEN}

Si bien Venezuela no es un caso representativo de nuevo régimen democrático, ya que ha funcionado ininterrumpidamente como tal desde hace treinta años, sí existen una serie de elementos que permiten plantear la existencia de una importante ruptura en la composición de la élite parlamentaria, constituyendo las dos últimas legislaturas un punto de inflexión claro en el desarrollo político venezolano y una muestra de la ruptura definitiva del bipartidismo que venía funcionando desde 1958.

El objeto de este artículo es el estudio de la estructura sociológica de la élite parlamentaria en el período 1993 1998, comparando algunas particularidades de la misma con características de la actual legislatura intentando establecer rupturas y continuidades entre ambas Cámaras. En el mismo se contrastan hipótesis clásicas, al estilo de las recogidas por Putnam y Von Beyme sobre la relación entre determinadas características sociodemográficas y demográficas y la élite política.

Palabras clave: Élite Parlamentarias - Venezuela.

\section{ABSTRACT}

Although Venezuela is not a representative case of new democracy, because it is established thirty years ago, there are now some important element that implied an break in parliamentarian elite composition. The last two legislatures can be interpreted as an evident point in Venezuela Political.

The object of this article is the study of sociological estructure of parliamentarian elite durign the period of 1993-1998, comparing some of their particularities and trying to stablish breaks and continuities along them. Some clasical hipothesis, as posited by Von Beyme and Putnam about correlation sociademoghapich characterists and political elites, are contrasted in the article.

Key words: Parliamentaris Elites - Venezuelan. 